TUMOUR METABOLISM

\title{
Integrating serine with tumour initiation
}

Although many tumours, including epidermal squamous cell carcinomas (SCCs), can arise from normal tissue stem cells (SCs), how SC metabolism might affect tumour initiation and growth remains unclear. Baksh et al. have delineated a pathway through which serine availability is integrated with SC fate decisions and can control SCC initiation.

Expression of the transcription factor SOX2 in keratin 14 positive $\left(\mathrm{K} 14^{+}\right)$epidermal SCs in mice can induce pre-malignant lesions. In order to look at early metabolic changes in these cells independent of hyperproliferation, the authors used SOX $2^{+}$epidermal SCs isolated from mice at postnatal day 4 , at which time these cells and wildtype (WT) epidermal SCs had similar rates of proliferation. Analysis of amino acid requirements of these cells indicated similar rates of consumption of all amino acids in $\mathrm{SOX}_{2}{ }^{+}$and WT epidermal SCs apart from serine, which was consumed at a higher rate by SOX2 ${ }^{+}$ epidermal SCs.

Withdrawal of extracellular serine (as well as glycine, which can be converted to serine) from the culture medium led to reduced intracellular levels of serine and slower proliferation in $\mathrm{SOX}_{2}{ }^{+}$epidermal SCs compared with WT cells that was attributed to the presence of serine alone (glycine withdrawal had no effect). The reduced serine levels and proliferation were surprising given that cells can synthesize serine de novo, and the authors found that the serine synthesis pathway (SSP), which produces serine from glucose, was blocked in $\mathrm{SOX}_{2}{ }^{+}$epidermal SCs.

Further analysis indicated that $\mathrm{SOX}^{+}$epidermal SCs were not defective in glucose metabolism more generally, but that they had a higher rate of pyruvate import and utilization in mitochondria, and several lines of evidence indicated that this impaired the SSP and increased dependence on extracellular serine. Loss of pyruvate from the cytosol resulted in a reduced $\mathrm{NAD}^{+}: \mathrm{NADH}$ ratio, as pyruvate is required for $\mathrm{NAD}^{+}$regeneration during glycolysis in the cytosol. $\mathrm{NAD}^{+}$is a co-factor for PHGDH, the rate-limiting enzyme in the SSP; consistent with a role for the reduced $\mathrm{NAD}^{+}: \mathrm{NADH}$ ratio in the SSP block observed in SOX $2^{+}$ epidermal SCs, several different methods of increasing the cytosolic $\mathrm{NAD}^{+}: \mathrm{NADH}$ ratio rescued the SSP deficiency.

The authors observed similar results with in vivo serine deprivation. Placing pregnant mice on a serine-free and glycine-free diet impaired proliferation of SOX $2^{+}$ epidermal SCs isolated from pups but had no effect on WT epidermal SCs. This could be rescued by increasing the $\mathrm{NAD}^{+}: \mathrm{NADH}$ ratio via expression of a bacterial NADH oxidase (LbNOX) in the mice.

Interestingly, dietary serine depletion increased differentiation of WT epidermal SCs but had no effect on differentiation of SOX2 ${ }^{+}$ epidermal SCs. This lack of differentiation was due to SSP deficiency in $\mathrm{SOX}^{+}$epidermal SCs. The authors found that exogenous supplementation of $\alpha$-ketoglutarate ( $\alpha \mathrm{KG})$, a by-product of the SSP, induced

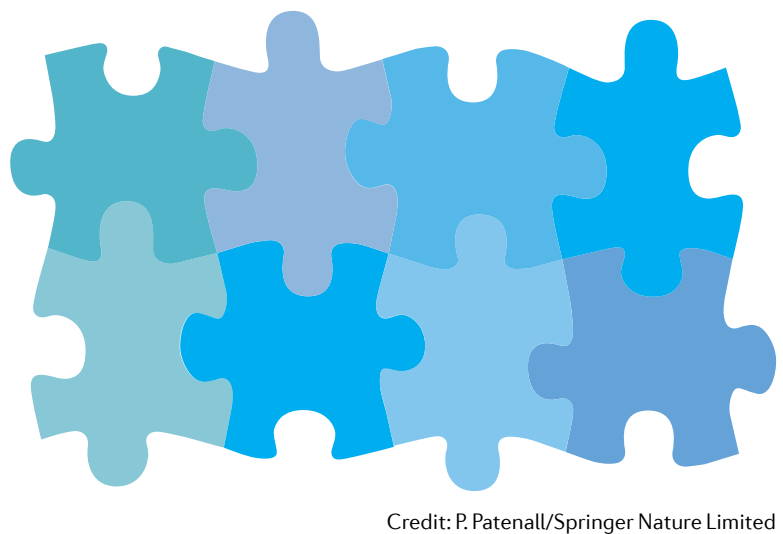

differentiation of these cells in vitro, as this bypasses the requirement for a functional SSP. In addition, exogenous aKG was sufficient to induce differentiation in WT epidermal SCs. One function of aKG is as a co-substrate for dioxygenases, and the authors attributed at least part of the effect on differentiation to $\alpha \mathrm{KG}$-dependent dioxygenases such as the Jumonjidomain family of histone demethylases that catalyse de-methylation of trimethylated histone $\mathrm{H} 3$ lysine 27 (H3K27me3). H3K27me3 normally represses the expression of genes involved in differentiation in the skin.

To examine how this pathway affects tumour initiation, the authors used DMBA-TPA chemical carcinogenesis in WT mice placed on a serine-free and glycine-free diet and found that this significantly impaired tumour initiation compared with mice receiving a normal diet. The tumours that did form in the absence of exogenous serine had reduced $\mathrm{H} 3 \mathrm{~K} 27 \mathrm{me} 3$ levels and increased differentiation, an effect that required an intact SSP, consistent with their earlier findings. Similar results were observed in xenografts of human SCC cell lines. Furthermore, increasing intracellular aKG levels by silencing expression of $\mathrm{OGDH}$, an enzyme that normally consumes $\alpha \mathrm{KG}$, was sufficient to block SCC xenograft growth and increase differentiation in mice fed a serine-free and glycine-free diet. Finally, H3K27me3 levels correlated with tumour grade in 139 primary human SCCs, indicating that this pathway likely operates in human tumours.

Overall, these data indicate that in the presence of serine starvation, the SSP can function as a tumour suppressor in skin SCC through aKG-dependent induction of differentiation, which depletes the epidermal SC pool. This study also suggests that serine depletion or inhibiting serine uptake might be used to eliminate pre-malignant SCs.

Sarah Seton-Rogers

ORIGINAL ARTICLE Baksh, S. C. et al.

Extracellular serine controls epidermal stem cell fate and tumour initiation. Nat. Cell Biol. https://

doi.org/10.1038/s41556-020-0525-9 (2020) 